Ultrahigh Energy Resolution Gamma-ray Spectrometers for Precision Measurements of Uranium Enrichment

S. Ali, I. D. Hau, T. R. Niedermayr, S. Friedrich

June 9, 2006

MARC VII (Methods \& Applications of Radioanalytical Chemistry)

Kona, , HI, United States

April 3, 2006 through April 7, 2006 
This document was prepared as an account of work sponsored by an agency of the United States Government. Neither the United States Government nor the University of California nor any of their employees, makes any warranty, express or implied, or assumes any legal liability or responsibility for the accuracy, completeness, or usefulness of any information, apparatus, product, or process disclosed, or represents that its use would not infringe privately owned rights. Reference herein to any specific commercial product, process, or service by trade name, trademark, manufacturer, or otherwise, does not necessarily constitute or imply its endorsement, recommendation, or favoring by the United States Government or the University of California. The views and opinions of authors expressed herein do not necessarily state or reflect those of the United States Government or the University of California, and shall not be used for advertising or product endorsement purposes. 


\section{Log Number :}

Title: Ultrahigh energy resolution Gamma-ray spectrometers for precision measurements of Uranium enrichment

Authors: Shafinaz Ali, I Dragos Hau, Thomas R. Niedermayr, Stephan Friedrich

Address: LLNL, Advanced Detector Group, 7000 East Ave, L-270, Livermore, CA 94550, USA

Corresponding Authors' Fax and E-mail: Shafinaz Ali, Fax (925) 424 5512, e-mail: ali2@1ln1.gov 


\title{
Ultrahigh energy resolution Gamma-ray spectrometers for precision measurements of Uranium enrichment
}

\author{
Shafinaz Ali \\ ali2@1lnl.gov \\ I Dragos Hau \\ Hau2@1ln1.gov \\ Thomas R. Niedermayr \\ Niedermayr1@1lnl.gov \\ Stephan Friedrich \\ Friedrich1@1lnl.gov
}

LLNL, Advanced Detector Group, 7000 East Ave, L-270, Livermore, CA 94550, USA

Key words: isotope analysis, uranium enrichment, cryogenic spectrometers, Gammaspectroscopy 


\title{
Ultrahigh energy resolution Gamma-ray spectrometers for precision measurements of Uranium enrichment
}

Shafinaz Ali, I Dragos Hau, Thomas R Niedermayr, Stephan Friedrich

LLNL, Advanced Detector Group, 7000 East Ave, L-270, Livermore, CA 94550, USA

\begin{abstract}
Superconducting Gamma-ray detectors offer an order of magnitude higher energy resolution than conventional high-purity germanium detectors. This can significantly increase the precision of non-destructive isotope analysis for nuclear samples where line overlap affects the errors of the measurement. We have developed Gamma-detectors based on superconducting molybdenum-copper sensors and bulk tin absorbers for nuclear science and national security applications. They have, depending on design, an energy resolution between $\sim 50$ and $\sim 150 \mathrm{eV} \mathrm{FWHM}$ at $\sim 100 \mathrm{keV}$. Here we apply this detector technology to the measurement of uranium isotope ratios, and discuss the trade-offs between energy resolution and quantum efficiency involved in detector design.
\end{abstract}

\section{Introduction}

Gamma spectroscopy is widely used to determine the isotopic composition of nuclear materials ${ }^{1}$. Upon decay, each isotope emits Gamma rays and X-rays with characteristic energies, and their intensity can be related to the isotope abundance. Highprecision isotope ratio measurements are typically based on emission lines with similar energies to reduce errors due to the energy dependence of self-absorption and detection efficiency. These lines often overlap with each other when analyzed with a conventional 
high-purity germanium (HPGe) spectrometer. This increases the statistical errors, and thus reduces the limiting precision of the measurement.

One important example is the measurement of uranium enrichment. For routine analysis, NaI detectors are typically used to infer the level of enrichment from the strength of the $186 \mathrm{keV}$ line of U-235 above the Compton background. If higher precision is required, HPGe detectors can be used to infer enrichment from the emission of the thorium daughters at $92 \mathrm{keV}$, since U-238 decays into Th-234 with Gamma emission lines at 92.4 and $92.8 \mathrm{keV}$, and the nearby $\mathrm{Th}_{\alpha 1} \mathrm{X}$-ray at $93.35 \mathrm{keV}$ originates

mostly from the decay of U-235 $2,3,4$. This approach works well with HPGe detectors for moderate levels of enrichment when the characteristic emission lines have roughly equal strength. Line overlap makes this approach less precise in cases of very high or very low levels of enrichment, when weak emission lines in close vicinity to strong ones have to be analyzed.

Superconducting Gamma-spectrometers can address the line overlap problem, since their energy resolution is not limited by the statistics of electron-hole pair generation. They can reduce statistical errors, and can thus make the $92 \mathrm{keV}$ region of the Gamma-spectrum more relevant for measuring uranium enrichment. Here we discuss the performance of superconducting Gamma-spectrometers, their relevance for measuring uranium enrichment, and the trade-offs involved in the detector design.

\section{Spectrometer}

Cryogenic gamma-ray microcalorimeters consist of a gamma-ray absorber attached to a highly sensitive thermometer. The thermometer often consists of a thin film 
superconductor, also known as a transition-edge sensor (TES), operated in the narrow temperature range of the transition from its superconducting to normal state. Here its resistance changes rapidly as a function of temperature so that the energy of a single gamma-photon can be determined with extremely high precision. The absorber and the thermometer are strongly thermally coupled together, while they are weakly coupled to the cold bath. In the simplest case ${ }^{5}$, random thermal fluctuations across this weak thermal conductance $G$ set the limit on the energy resolution of microcalorimeters to $\Delta E_{F H W M} \approx 2.355 \zeta \sqrt{k_{B} T^{2} C_{a b s}}$, where, $\zeta$ is a factor of order 1-2 that depends on the thermal properties of the thermometer, $\mathrm{T}$ is the absolute temperature and $\mathrm{C}_{\mathrm{abs}}$ is the heat capacity of the absorber. For high energy resolution, the operating temperature $\mathrm{T}$ and the heat capacity $\mathrm{C}_{\mathrm{abs}}$ of the microcalorimeter must therefore be as small as feasible. For practical applications, an operating temperature of $0.1 \mathrm{~K}$ and absorber volume $\sim \mathrm{mm}^{3}$ offer a compromise between ease of operation and performance. This produces, depending on material choices and absorber sizes, cryogenic spectrometers with energy resolution between $50-150 \mathrm{eV}$ FWHM with quantum efficiency around 50\% at $100 \mathrm{keV}$.

At LLNL, we are developing cryogenic gamma ray spectrometers based on bulk Sn absorber and $\mathrm{Mo} / \mathrm{Cu}$ multilayer transition-edge sensors ${ }^{6,7,8}$. The TES thermistors are made photolithographically on $4 " \mathrm{Si}$ wafers, and a $\sim \mathrm{mm}^{3} \mathrm{Sn}$ absorber is glued to each sensor with stycast epoxy. The spectrometer is cooled to its operating temperature of $0.1 \mathrm{~K}$ in a two-stage adiabatic demagnetization refrigerator $(\mathrm{ADR})^{9,10}$. The detector is voltage biased at the onset of its superconducting-to-normal transition and exposed to radiation. The current signal from the spectrometer is readout by a low noise dc superconducting quantum interference device (SQUID) current amplifier. The gamma 
induced pulse signals are further amplified at room temperature and digitized by a 14-bit data acquisition system. The data are subsequently optimally filtered to extract spectral information.

\section{Simulations}

A Monte-Carlo N-Particle (MCNP) simulation was performed to estimate the performance of the cryogenic gamma-ray spectrometer in measuring ${ }^{235} \mathrm{U}$ enrichment. The response of a cryogenic detector with a $1 \mathrm{~mm}^{3} \mathrm{Sn}$-absorber and with an energy resolution of $100 \mathrm{eV}$ FWHM was compared to that of an HPGe detector with an energy resolution of $500 \mathrm{eV}$ FWHM for a natural uranium sample (figure 1). The ${ }^{234} \mathrm{Th}$ gamma lines at $92.38 \mathrm{keV}$ and $92.80 \mathrm{keV}$ and the $\mathrm{Th} \mathrm{K} \alpha_{1} \mathrm{x}$-ray at $93.35 \mathrm{keV}$ are fully resolved by the cryogenic spectrometer with $100 \mathrm{eV}$ FWHM, while they blend together when using an HPGe detector. This illustrates the potential of high-resolution cryogenic spectrometers to determine isotope ratios, provided the total number of counts is sufficient for good statistics.

To optimize the detector design and trade-offs in energy resolution and quantum efficiency, we can quantify the statistical error in measuring enrichment as a function of energy resolution. For this, we consider the case when two emission lines with intensities $I_{1}$ and $I_{2}$ at energies $E_{1}$ and $E_{2}$ are examined with a spectrometer with an energy resolution $\Delta \mathrm{E}_{\mathrm{FWHM}}$. We assume that the spectrometer response can be described by a Gaussian function and that the Compton background B is constant over the energy range of interest. In this case, the statistical error $\sigma_{1} / I_{1}$ measurement can be calculated analytically ${ }^{10,11}$. Figure 2 shows this limiting error as a function of detector resolution $\Delta \mathrm{E}_{\mathrm{FWHM}}$ for a given line separation $\mathrm{E}_{1}-\mathrm{E}_{2}=550 \mathrm{eV}$ and $\mathrm{I}_{1} / \mathrm{I}_{2}=1 \%$ for different number of 
total counts $I_{\text {total }}=I_{1}+I_{2}$. As expected, the percent statistical error decreases with decreasing $\Delta \mathrm{E}_{\mathrm{FWHM}}$ rather sharply as long as there is line overlap, but then levels off as the detector resolution is sufficient to fully separate the lines of interest, with the remaining reduction in error being due to a better discrimination of the signal from the Compton background $\mathrm{B}$. We see that a detector with energy resolution of $\sim 300 \mathrm{eV}$ FWHM is already sufficient to fully resolve the $\mathrm{Th} \mathrm{K} \alpha_{1}$ line at $\mathrm{E}_{1}=93.35 \mathrm{keV}$ from the ${ }^{234} \mathrm{Th}$ gamma line at $\mathrm{E}_{2}=92.8 \mathrm{keV}$, at which point the precision of the enrichment measurement then depends mostly on the total number of counts and approaches $\sigma_{1} \approx \sqrt{I_{1}}$ as expected.

\section{Results}

We have measured a weakly radioactive $(20 \mathrm{nCi})$ low-enriched uranium sample to evaluate the performance of our cryogenic gamma ray spectrometer to determine ${ }^{235} \mathrm{U}$ enrichment using Th $\mathrm{K} \alpha_{1}$ at $93.35 \mathrm{keV}$ as a measure of ${ }^{235} \mathrm{U}$ and the ${ }^{234} \mathrm{Th}$ gamma lines at 92.38 and $92.80 \mathrm{keV}$ as a measure of ${ }^{238} \mathrm{U}$ (figure 3). This particular cryogenic detector has an energy resolution of $90 \mathrm{eV} \mathrm{FWHM}$ and can thus well resolve the relevant gamma and x-ray lines. Also, the Compton background count is low and the assumption that the detector response can be characterized by a Gaussian function is justified. For comparison, we include a measurement of the same sample with a planar HPGe spectrometer, taken over the same acquisition time of $\sim 3$ days as the data from the single pixel cryogenic spectrometer.

The isotopic abundances of ${ }^{235} \mathrm{U}\left(\mathrm{A}_{235}\right)$ and ${ }^{238} \mathrm{U}\left(\mathrm{A}_{238}\right)$ are extracted from the observed peak intensities $\mathrm{I}_{1}\left(\mathrm{Th} \mathrm{K} \alpha_{1}\right)$ and $\mathrm{I}_{2}\left({ }^{234} \mathrm{Th}\right)$ by $\frac{A_{235}}{A_{238}}=\frac{I_{1}}{I_{2}} \frac{T_{1}}{T_{2}} \frac{\eta_{2}}{\eta_{1}} \frac{B_{2}}{B_{1}}$, where $\mathrm{T}_{1,2}$ are 
the half-lives, $\eta_{1,2}$ are detection efficiencies and $B_{1,2}$ are the branching ratios. Since the two lines are closely spaced in energy, we can assume $\eta_{1} / \eta_{2}=1$. Note that the literature values for branching ratios for the gamma and $\mathrm{x}$-ray lines in the $90-100 \mathrm{keV}$ region of $\mathrm{U}$ spectrum quoted in the literature vary significantly ${ }^{4}$. In this paper we use $0.0260,0.02560$ and 0.0550 as the branching ratios for the emission lines at $92.38,92.790 \mathrm{keV}$ and 93.356 $\mathrm{keV}$, respectively, since these values are used by the $\mathrm{U} 235$ analysis code developed at LLNL for measuring uranium enrichment ${ }^{3}$.

We determine the ${ }^{235} \mathrm{U}$ enrichment of this sample to be $(1.14 \pm 0.14) \%$. For comparison, the enrichment value extracted with the U235 code from the HPGe spectrum is $(1.017 \pm 0.014) \%$. This indicates that a single pixel cryogenic gamma spectrometer is not sufficient to improve the precision of the measurement, because of its small detection efficiency, despite the exquisitely high-energy resolution. This is due to the fact that the line overlap problem of HPGe detector is compensated for by the increased detector efficiency they offer, especially, when analyzing weak radioactive samples as in this experiment.

\section{Discussion}

The low number of counts in the weak $93.35 \mathrm{keV}$ x-ray line limits the precision of ${ }^{235} \mathrm{U}$ enrichment measurement for a single pixel cryogenic spectrometer. However, the statistical error can be reduced by increasing absorber volume thus the quantum efficiency of each pixel and by fabricating large detector arrays. For example, for uranium analysis, increasing the size of each individual absorber by a factor of 10 could increase the number of counts. Although this will degrade energy resolution by a factor 
of $\sim \sqrt{10}$, it will improve precision of enrichment measurement as long as there is no line overlap (cf. figure2).

In addition, a spectrometer composed of a 100-pixel array, where each pixel retains the high energy resolution will increase the total number of count by hundred fold and reduce the statistical error by a factor of $\sim 10$. We are currently building such a multipixel cryogenic spectrometer ${ }^{12}$. It will reduce the statistical error by a factor of $\sim 30$ and improve the statistical precision of enrichment measurement to $\sim 0.001 \%$.

\section{Conclusions}

Cryogenic Gamma-ray spectrometers offer higher energy resolution than conventional HPGe spectrometer. This can, for example, improve the measurement of uranium using the $92 \mathrm{keV}$ region, provided the total numbers of counts in the lines of interest are sufficient. We have built a superconducting gamma ray spectrometer based on $\mathrm{Sn}$ absorbers and $\mathrm{Mo} / \mathrm{Cu}$ multilayer sensors with an energy resolution of $90 \mathrm{eV}$ FWHM at $\sim 100 \mathrm{keV}$. We have used it to analyze a low-enriched uranium sample and find for this application, the highest signal-to-noise ratio is achieved with energy resolution $300 \mathrm{eV}$ FWHM. Note that for other applications such as Pu isotope analysis much higher energy resolution is needed ${ }^{13}$. Larger absorber sizes and arrays of 100 pixels are needed to improve the precision of the measurement to $\sim 0.001 \%$.

\section{Acknowledgments}

We gratefully acknowledge the support of the U.S. Department of Energy, Office of Non-Proliferation Research and Engineering, NA-22. This work was performed under 
the auspices of the U.S. Department of Energy by University of California Lawrence Livermore National Laboratory under contract No. W-7405-Eng-48.

\section{References}

1. D. REILLY, N. ENSSLIN, H. SMITH, JR., S. KRIENER, Passive Non-Destructive Assay of Nuclear Materials, Office of Nuclear Regulatory Research (NUREG/CR-5550), Washington, 1991.

2. T.N. DRAGNEV, B.P. DAMYANOV, Proc. IAEA Symposium on Nuclear Material Safeguards, I (1978) 739.

3. T.N. DRAGNEV, B.P. DAMYANOV, G.G. Grozev, Proc. IAEA Symposium on Nuclear Material Safeguards, II (1982) 258.

4. D. CLARK, U235: A Gamma Ray Analysis Code for Uranium Isotopic Determination, Lawrence Livermore National Laboratory, UCRL-ID-125727 (1996).

5. S.H. MOSELEY, J.C. MATHER, D. MCCAMMON, Journal of Applied Physics, 56 (1984) 1257.

6. S.E. LABOV, M. FRANK, J.B. LE GRAND, M.A. LINDEMAN, H.NETEL, L,J, HILLER, D. CHOW, S. FRIEDRICH, C.A. MEARS, G.CALDARA, A.T.

BARKFKNECHT, Proc. $7^{\text {th }}$ Int. Workshop on Low Temperature Detectors, (1997) 82.

7. M. F. CUNNINGHAM, J.N. ULLOM, T.MIAZAKI, S.E. LABOV, J.CLARKE, T.M. LANTING, A.T. LEE, P.L. RICHARDS, J. YOON, H. SPIELER, Appl. Physics Lett., 66 (2002) 159.

8. S.F. TERRACOL, S. ALI, T.N. NIEDERMAYR, I.D. HAU, O.B. DRURY, Z.A. ALI, T. MIYAZAKI, M.F. CUNNINGHAM, J.G. DREYER, J.D. LEACOCK, 
S.FRIEDRICH, 2004 IEEE Nuclear Science Symposium Conference Record, (2004) 1006.

9. C. HAGMANN, P.L. RICHARDS, Cryogenics, 34 (1994) 221.

10. P.L. RYDER, Scanning Electron Microscopy, 1 (1977) 273.

11. O.B. DRURY, S.F. TERRACOL, S. FRIEDRICH, Phys. Status Solidi C, 2 (2005) 1468.

12. S. FRIEDRICH, S.F. TERRACOL, T. MIYAJAKI, O.B. DRURY, Z.A ALI, M.F. CUNNINGHAM, T. R. NIEDERMAYR, T.W. BARBEE JR., J.D. BATTEUX, S.E.

LABOV, Proc. SPIE, 5540 (2004) 156.

13. S. ALI, S.F. TERRACOL, I.D. HAU, O.B. DRURY, S. FRIEDRICH, Proc. $46^{\text {th }}$ Ann. Mtg. INMM, (2005). 


\section{List of Figures:}

Figure 1: MCNP simulations of an HPGe spectrometer with $500 \mathrm{eV}$ FWHM and a cryogenic spectrometer with $100 \mathrm{eV}$ FWHM. The geometry used in the simulation does not take into account all surrounding materials and thus underestimates the Compton background. Note the increased number of escape lines due to the small pixel size of cryogenic detector. However, since these lines can be fully resolved, they do not affect the precision of the measurement.

Figure 2: Statistical error for measuring a line at energy $E_{1}$ with intensity $I_{1}$ in the presence of a much stronger line at $\mathrm{E}_{2}$ with intensity $\mathrm{I}_{2}=100 \mathrm{I}_{1}$ as a function of detector energy resolution $\Delta \mathrm{E}_{\mathrm{FWHM}}$. The simulation assumes an energy separation of $\mathrm{E}_{1}-\mathrm{E}_{2}=550$ $\mathrm{eV}$, corresponding to the separation of ${ }^{234} \mathrm{Th}$ line at $92.80 \mathrm{keV}$ and the $\mathrm{Th} \mathrm{K}_{1}$ at 93.35 $\mathrm{keV}$, and a constant Compton background $\mathrm{B}$. As expected, the relative statistical error decreases with improved $\Delta \mathrm{E}_{\mathrm{FWHM}}$ rather sharply as long as there is line overlap, but then levels off as the detector resolution is sufficient to fully separate the lines of interest, with the remaining reduction in error being due to a better discrimination of the signal from the Compton background B. For well-separated lines the precision of the enrichment measurement depends mostly on the total number of counts and approaches $\sigma_{1} \approx \sqrt{I_{1}}$.

Figure 3: Gamma spectra of a low-enriched uranium sample taken with a cryogenic spectrometer with an energy resolution of $90 \mathrm{eV}$ FWHM and a planar HPGe spectrometer with an energy resolution of $600 \mathrm{eV}$ FWHM. The emission lines at $92.38 \mathrm{keV}$ and 92.80 $\mathrm{keV}$ and at $93.35 \mathrm{keV}$, which are a measure of ${ }^{238} \mathrm{U}$ and ${ }^{235} \mathrm{U}$ concentration respectively, are fully resolved by the cryogenic detector. 
Figure 1

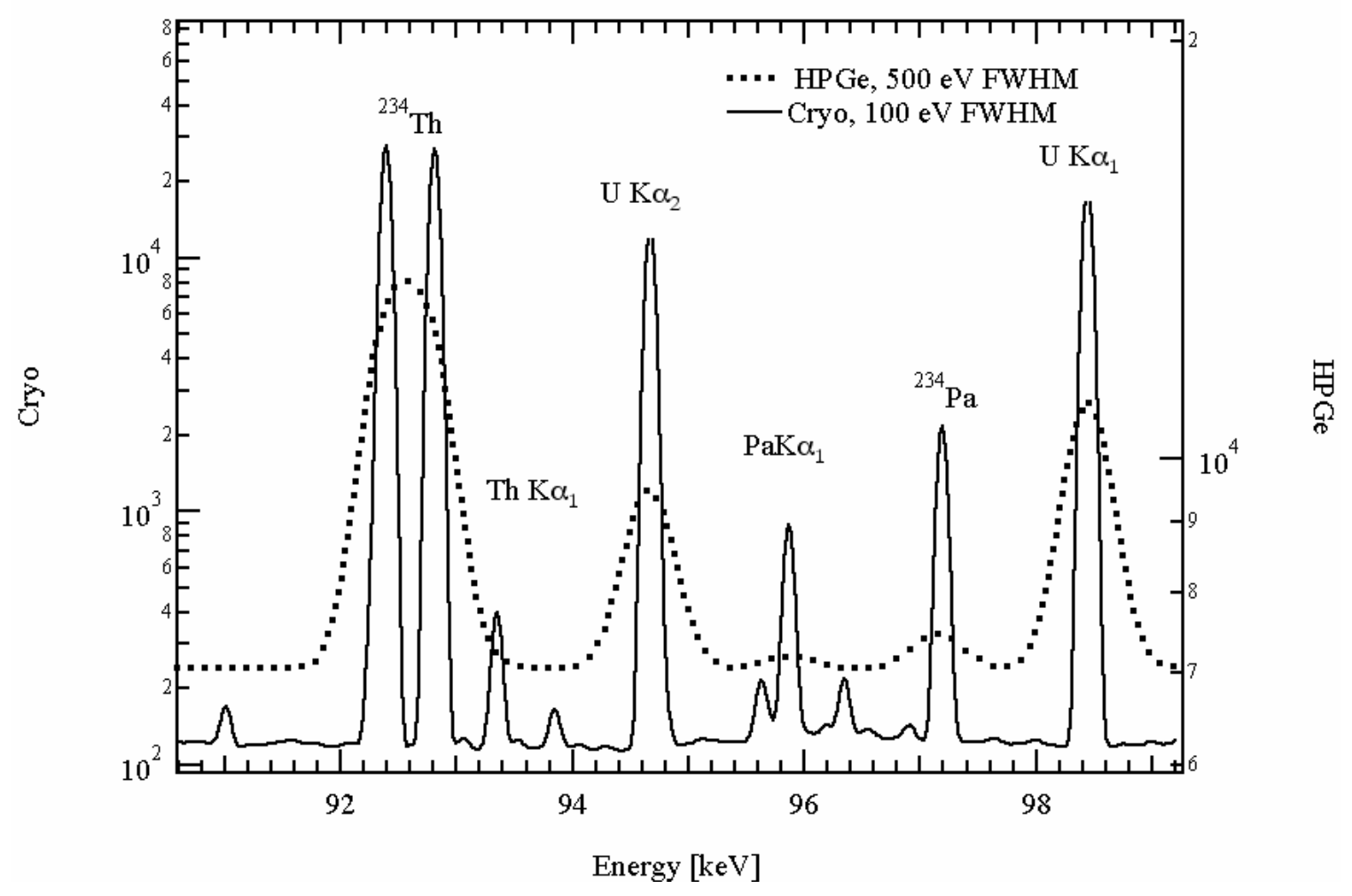


Figure 2

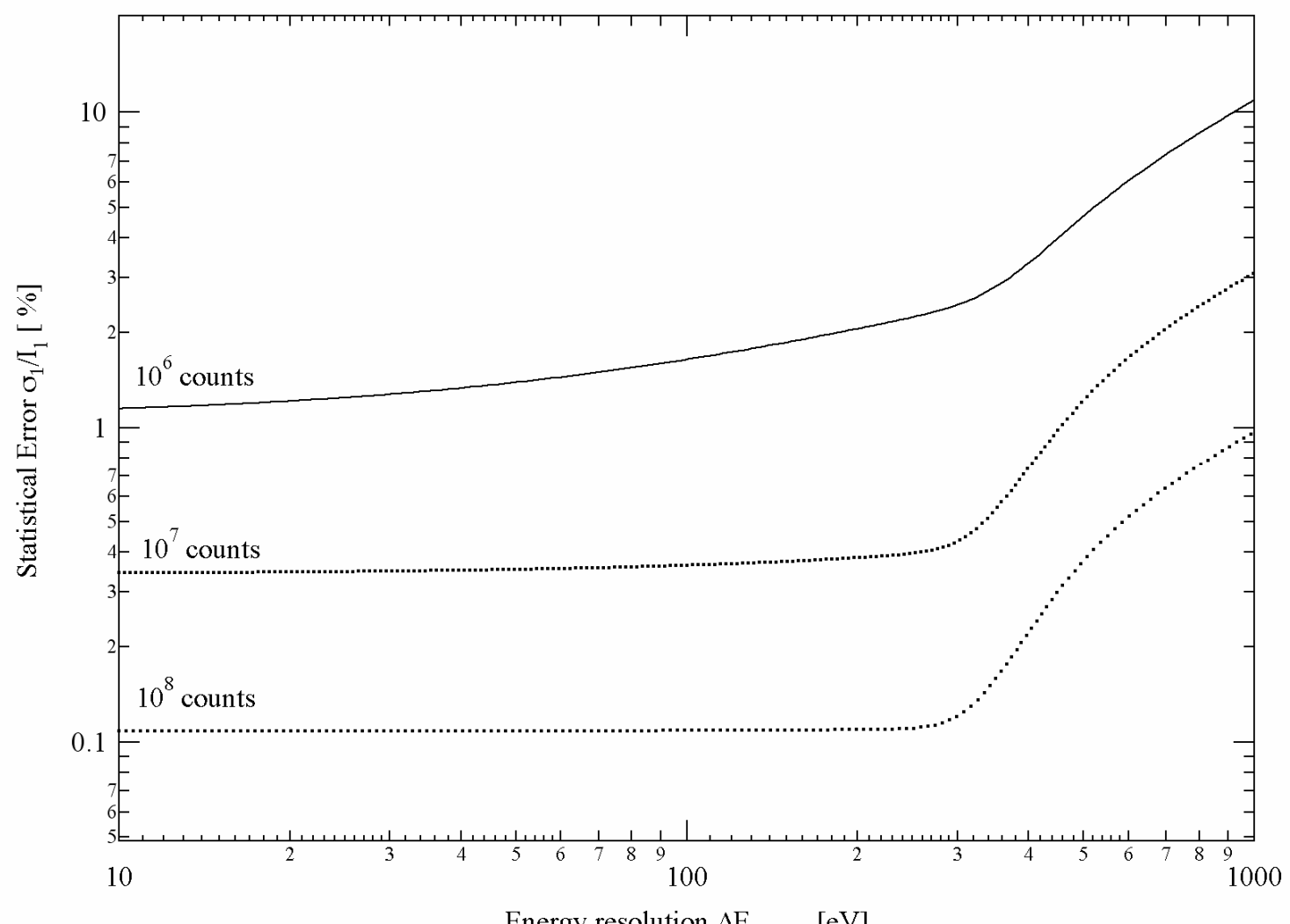

Energy resolution $\Delta \mathrm{E}_{\mathrm{FWHM}}[\mathrm{eV}]$ 
Figure 3

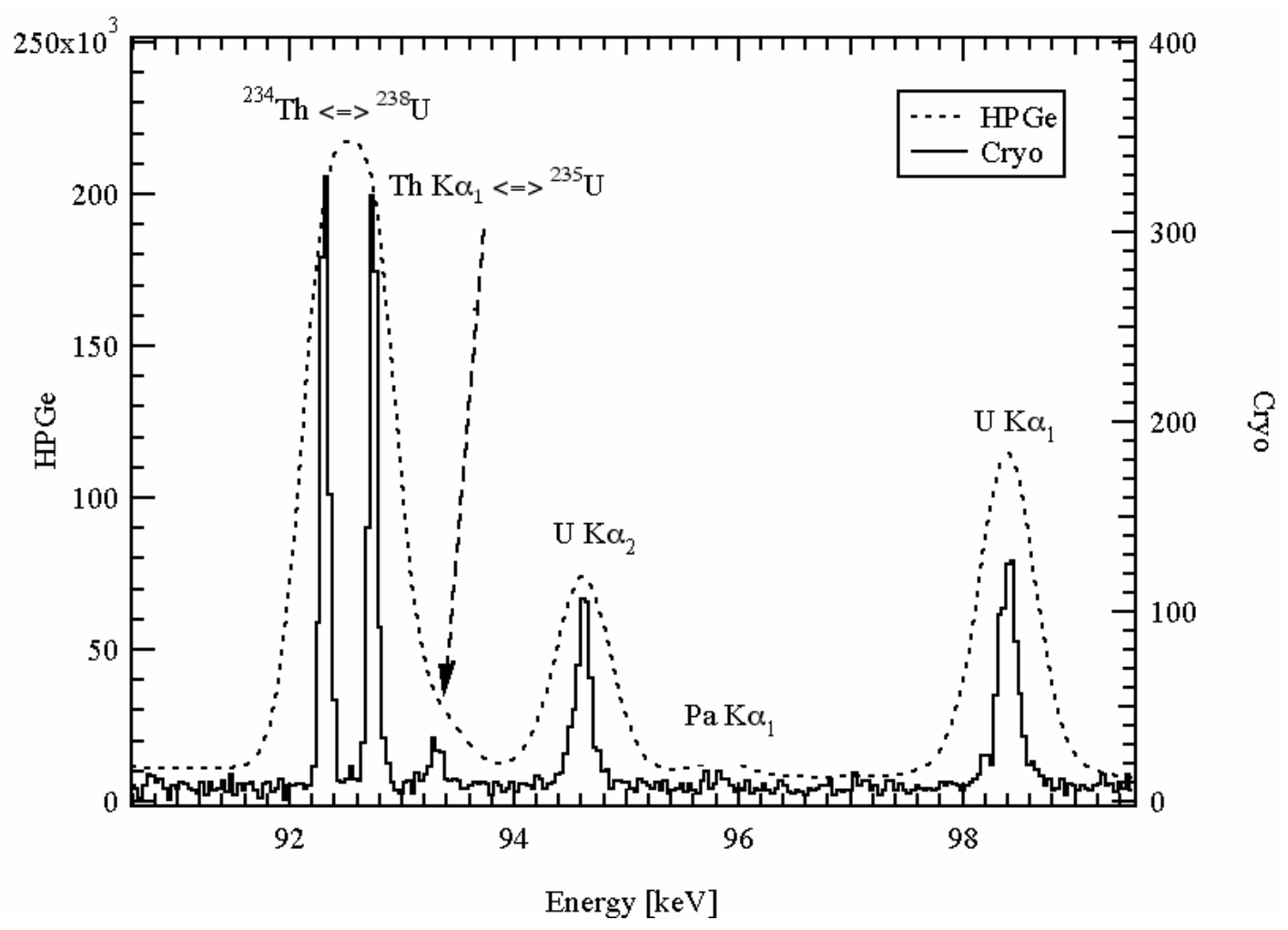

